\title{
The biHecke monoid of a finite Coxeter group
}

\author{
Florent Hivert $^{1}$, Anne Schilling ${ }^{2}$, and Nicolas M. Thiéry ${ }^{2,3}$ \\ ${ }^{1}$ LITIS (EA 4108), Université de Rouen, Avenue de l'Université BP12 76801 Saint-Etienne du Rouvray, France and \\ Institut Gaspard Monge (UMR 8049) \\ ${ }^{2}$ Department of Mathematics, University of California, One Shields Avenue, Davis, CA 95616, U.S.A. \\ ${ }^{3}$ Univ Paris-Sud, Laboratoire de Mathématiques d'Orsay, Orsay, F-91405; CNRS, Orsay, F-91405, France
}

\begin{abstract}
For any finite Coxeter group $W$, we introduce two new objects: its cutting poset and its biHecke monoid. The cutting poset, constructed using a generalization of the notion of blocks in permutation matrices, almost forms a lattice on $W$. The construction of the biHecke monoid relies on the usual combinatorial model for the 0-Hecke algebra $H_{0}(W)$, that is, for the symmetric group, the algebra (or monoid) generated by the elementary bubble sort operators. The authors previously introduced the Hecke group algebra, constructed as the algebra generated simultaneously by the bubble sort and antisort operators, and described its representation theory. In this paper, we consider instead the monoid generated by these operators. We prove that it admits $|W|$ simple and projective modules. In order to construct the simple modules, we introduce for each $w \in W$ a combinatorial module $T_{w}$ whose support is the interval $[1, w]_{R}$ in right weak order. This module yields an algebra, whose representation theory generalizes that of the Hecke group algebra, with the combinatorics of descents replaced by that of blocks and of the cutting poset.

Résumé. Pour tout groupe de Coxeter fini $W$, nous définissons deux nouveaux objets: son ordre de coupures et son monoïde de Hecke double. L'ordre de coupures, construit au moyen d'une généralisation de la notion de bloc dans les matrices de permutations, est presque un treillis sur $W$. La construction du monoïde de Hecke double s'appuie sur le modèle combinatoire usuel de la 0 -algèbre de Hecke $H_{0}(W)$ i.e., pour le groupe symétrique, l'algèbre (ou le monoïde) engendré par les opérateurs de tri par bulles élémentaires. Les auteurs ont introduit précédemment l'algèbre de Hecke-groupe, construite comme l'algèbre engendrée conjointement par les opérateurs de tri et d'anti-tri, et décrit sa théorie des représentations. Dans cet article, nous considérons le monoïde engendré par ces opérateurs. Nous montrons qu'il admet $|W|$ modules simples et projectifs. Afin de construire ses modules simples, nous introduisons pour tout $w \in W$ un module combinatoire $T_{w}$ dont le support est l'intervalle $[1, w]_{R}$ pour l'ordre faible droit. Ce module détermine une algèbre dont la théorie des représentations généralise celle de l'algèbre de Hecke groupe, en remplaçant la combinatoire des descentes par celle des blocs et de l'ordre de coupures.
\end{abstract}

Keywords: Coxeter groups, Hecke algebras, representation theory, blocks of permutation matrices

\section{Introduction}

The usual combinatorial model for the 0-Hecke algebra $H_{0}\left(\mathfrak{S}_{n}\right)$ of the symmetric group is the algebra (or monoid) generated by the (anti) bubble sort operators $\pi_{1}, \ldots, \pi_{n-1}$, where $\pi_{i}$ acts on words of length $n$ and sorts the letters in positions $i$ and $i+1$ decreasingly. By symmetry, one can also construct the bubble sort operators $\bar{\pi}_{1}, \ldots, \bar{\pi}_{n-1}$, where $\bar{\pi}_{i}$ acts by sorting increasingly, and this gives an isomorphic 
construction $\bar{H}_{0}$ of the 0 -Hecke algebra. This construction generalizes naturally to any finite Coxeter group $W$. Furthermore, when $W$ is a Weyl group, and hence can be affinized, there is an additional operator $\pi_{0}$ projecting along the highest root.

In [HT09] the first and last author constructed the Hecke group algebra $\mathcal{H W}$ by gluing together the 0Hecke algebra and the group algebra of $W$ along their right regular representation. Alternatively, $\mathcal{H} W$ can be constructed as the biHecke algebra of $W$, by gluing together the two realizations $H_{0}(W)$ and $\bar{H}_{0}(W)$ of the 0 -Hecke algebra. $\mathcal{H W}$ admits a more conceptual description as the algebra of all operators on $\mathbb{K} . W$ preserving left antisymmetries; the representation theory of $\mathcal{H} W$ follows, governed by the combinatorics of descents. In [HST09], the authors further proved that, when $W$ is a Weyl group, $\mathcal{H} W$ is a natural quotient of the affine Hecke algebra.

In this paper, following a suggestion of Alain Lascoux, we study the biHecke monoid $M(W)$, obtained by gluing together the two 0-Hecke monoids. This involves the combinatorics of the usual poset structures on $W$ (left, right, left-right, Bruhat order), as well as a new one, the cutting poset, which in type $A$ is related to blocks in permutation matrices. The guiding principle is the use of representation theory to derive a (so far elusive) summation formula for the size of this monoid, using that the simple and projective modules of $M$ are indexed by the elements of $W$.

In type $A$, the tower of algebras $\left(\mathbb{K}\left[M\left(\mathfrak{S}_{n}\right)\right]\right)_{n \in \mathbb{N}}$ possesses long sought-after properties. Indeed, it is well-known that several combinatorial Hopf algebras arise as Grothendieck rings of towers of algebras. The prototypical example is the tower of algebras of the symmetric groups which gives rise to the Hopf algebra Sym of symmetric functions, on the Schur basis. Another example, due to Krob and Thibon [KT97], is the tower of the 0-Hecke algebras of the symmetric groups which gives rise to the Hopf algebra QSym of quasi-symmetric functions of [Ges84], on the $F_{I}$ basis. The product rule on the $F_{I}$ 's is naturally lifted through the descent map to a product on permutations, leading to the Hopf algebra FQSym of free quasi-symmetric functions. This calls for the existence of a tower of algebras $\left(A_{n}\right)_{n \in \mathbb{N}}$, such that each $A_{n}$ contains $H_{0}\left(\mathfrak{S}_{n}\right)$ and has its simple modules indexed by the elements of $\mathfrak{S}_{n}$. The biHecke monoids $M\left(\mathfrak{S}_{n}\right)$ and their Borel submonoids $M_{1}\left(\mathfrak{S}_{n}\right)$ satisfy these properties, and are therefore expected to yield new representation theoretical interpretations of the bases of FQSym.

In the remainder of this introduction, we briefly review Coxeter groups and their 0-Hecke monoids, and introduce our main objects of study: the biHecke monoids.

\subsection{Coxeter groups}

Let $(W, S)$ be a Coxeter group, that is, a group $W$ with a presentation

$$
W=\left\langle S \mid\left(s s^{\prime}\right)^{m\left(s, s^{\prime}\right)}, \forall s, s^{\prime} \in S\right\rangle,
$$

with $m\left(s, s^{\prime}\right) \in\{1,2, \ldots, \infty\}$ and $m(s, s)=1$. The elements $s \in S$ are called simple reflections, and the relations can be rewritten as $s^{2}=1$, where 1 is the identity in $W$ and:

$$
\underbrace{s s^{\prime} s s^{\prime} s \cdots}_{m\left(s, s^{\prime}\right)}=\underbrace{s^{\prime} s s^{\prime} s s^{\prime} \cdots}_{m\left(s, s^{\prime}\right)} \quad \text { for all } s, s^{\prime} \in S .
$$

Most of the time, we just write $W$ for $(W, S)$. In general, we follow the notation of [BB05], and we refer to this monograph and to [Hum90] for details on Coxeter groups and their Hecke algebras. Unless stated otherwise, we always assume that $W$ is finite, and denote its generators by $S=\left(s_{i}\right)_{i \in I}$, where $I=\{1,2, \ldots, n\}$ is the index set of $W$. 
The prototypical example is the Coxeter group of type $A_{n-1}$ which is the $n$-th symmetric group $(W, S):=\left(\mathfrak{S}_{n},\left\{s_{1}, \ldots, s_{n-1}\right\}\right)$, where $s_{i}$ denotes the elementary transposition which exchanges $i$ and $i+1$. The relations are $s_{i}^{2}=1$ for $1 \leq i \leq n-1$ and the braid relations:

$$
\begin{aligned}
s_{i} s_{j} & =s_{j} s_{i}, & & \text { for }|i-j| \geq 2, \\
s_{i} s_{i+1} s_{i} & =s_{i+1} s_{i} s_{i+1}, & & \text { for } 1 \leq i \leq n-2 .
\end{aligned}
$$

When writing a permutation $\mu \in \mathfrak{S}_{n}$ explicitly, we use the one-line notation, that is the sequence $\mu_{1} \mu_{2} \cdots \mu_{n}$, where $\mu_{i}:=\mu(i)$.

A reduced word $i_{1} \ldots i_{k}$ for an element $\mu \in W$ corresponds to a decomposition $\mu=s_{i_{1}} \cdots s_{i_{k}}$ of $\mu$ into a product of generators in $S$ of minimal length $k=\ell(\mu)$. A (right) descent of $\mu$ is an element $i \in I$ such that $\ell\left(\mu s_{i}\right)<\ell(\mu)$. If $\mu$ is a permutation, this translates into $\mu_{i}>\mu_{i+1}$. Left descents are defined analogously. The sets of left and right descents of $\mu$ are denoted by $\mathrm{D}_{L}(\mu)$ and $\mathrm{D}_{R}(\mu)$, respectively.

A Coxeter group $W$ comes equipped with four natural graded poset structures. Namely $\mu<\nu$ in Bruhat order (resp. left (weak), right (weak), left-right (weak) order) if some reduced word for $\mu$ is a subword (resp. right factor, left factor, factor) of some reduced word for $\nu$. In type $A$, the left (resp. right) order give the usual left (resp. right) permutahedron.

For $J \subseteq I$, we denote by $W_{J}=\left\langle s_{j} \mid j \in J\right\rangle$ the subgroup of $W$ generated by $s_{j}$ with $j \in J$. Furthermore, the longest element in $W_{J}$ (resp. $W$ ) is denoted by $s_{J}$ (resp. $w_{0}$ ).

\subsection{The 0-Hecke monoid}

The 0-Hecke monoid $H_{0}(W)=\left\langle\pi_{i} \mid i \in I\right\rangle$ of a Coxeter group $W$ is generated by the simple projections $\pi_{i}$ with relations $\pi_{i}^{2}=\pi_{i}$ for $i \in I$ and

$$
\underbrace{\pi_{i} \pi_{j} \pi_{i} \pi_{j} \cdots}_{m\left(s_{i}, s_{j}\right)}=\underbrace{\pi_{j} \pi_{i} \pi_{j} \pi_{i} \cdots}_{m\left(s_{i}, s_{j}\right)} \quad \text { for all } i, j \in I
$$

Thanks to these relations, the elements of $H_{0}(W)$ are canonically indexed by the elements of $W$ by setting $\pi_{w}:=\pi_{i_{1}} \cdots \pi_{i_{k}}$ for any reduced word $i_{1} \ldots i_{k}$ of $w$. We further denote by $\pi_{J}$ the longest element of the parabolic submonoid $H_{0}\left(W_{J}\right):=\left\langle\pi_{i} \mid i \in J\right\rangle$.

The right regular representation of $H_{0}(W)$ induces a concrete realization of $H_{0}(W)$ as a monoid of operators acting on $W$, with generators $\pi_{1}, \ldots, \pi_{n}$ defined by:

$$
w . \pi_{i}:= \begin{cases}w & \text { if } i \in \mathrm{D}_{R}(w), \\ w s_{i} & \text { otherwise. }\end{cases}
$$

In type $A, \pi_{i}$ sorts the letters at positions $i$ and $i+1$ decreasingly, and for any permutation $w, w \cdot \pi_{w_{0}}=$ $n \cdots 21$. This justifies naming $\pi_{i}$ an elementary bubble antisorting operator.

Another concrete realization of $H_{0}(W)$ can be obtained by considering instead the elementary bubble sorting operators $\bar{\pi}_{1}, \ldots, \bar{\pi}_{n}$, whose action on $W$ are defined by:

$$
w \cdot \bar{\pi}_{i}:= \begin{cases}w s_{i} & \text { if } i \in \mathrm{D}_{R}(w), \\ w & \text { otherwise. }\end{cases}
$$

In type $A$, and for any permutation $w$, one has $w \cdot \bar{\pi}_{w_{0}}=12 \cdots n$. 


\subsection{The biHecke monoid}

Definition 1.1 Let Wbe a finite Coxeter group. The biHecke monoid is the submonoid of functions from $W$ to $W$ generated simultaneously by the elementary bubble sorting and antisorting operators of (5) and (6):

$$
M:=M(W):=\left\langle\pi_{1}, \pi_{2}, \ldots, \pi_{n}, \bar{\pi}_{1}, \bar{\pi}_{2}, \ldots, \bar{\pi}_{n}\right\rangle .
$$

\subsection{Outline}

The remainder of this paper is organized as follows. In Section 2, we generalize the notion of blocks of permutation matrices to any Coxeter group, and use it to define a new poset structure on $W$, which we call the cutting poset; we prove that it is (almost) a lattice, and derive that its Möbius function is essentially that of the hypercube.

In Section 3, we study the combinatorial properties of $M(W)$. In particular, we prove that it preserves left and Bruhat order, derive consequences on the fibers and image set of its elements, prove that it is aperiodic, and study its conjugacy classes of idempotents.

In Section 4, our strategy is to consider a "Borel" triangular submonoid of $M(W)$ whose representation theory is simpler, but with the same number of simple modules, in the hope to later induce back information about the representation theory of $M(W)$. Namely, we study the submonoid $M_{1}(W)$ of the elements fixing 1 in $M(W)$. This monoid not only preserves Bruhat order, but furthermore is contracting. It follows that it is $\mathcal{J}$-trivial which is the desired triangularity property. It is for example easily derived that $M_{1}(W)$ has $|W|$ simple modules, all of dimension 1. In fact most of our results about $M_{1}$ generalize to any $\mathcal{J}$-trivial monoid, which is the topic of a separate paper on the representation theory of $\mathcal{J}$-trivial monoids [DHST10].

In Section 5, we construct, for each $w \in W$, the translation module $T_{w}$ by induction of the corresponding simple $M_{1}$-module. It is a quotient of the indecomposable projective module $P_{w}$ of $M(W)$, and therefore admits the simple module $S_{w}$ of $M(W)$ as top. It further admits a simple combinatorial model with the interval $[1, w]_{R}$ as support, and which passes down to $S_{w}$. We derive a formula for the dimension of $S_{w}$, using an inclusion-exclusion on the sizes of intervals in $\left(W, \leq_{R}\right)$, along the cutting poset. On the way, we study the algebra $\mathcal{H} W^{(w)}$ induced by the action of $M(W)$ on $T_{w}$. It turns out to be a natural $w$-analogue of the Hecke group algebra, acting not anymore on the full Coxeter group, but on the interval $[1, w]_{R}$ in right order. All the properties of the Hecke group algebra pass through this generalization, with the combinatorics of descents being replaced by that of blocks and of the cutting poset. In particular, $\mathcal{H} W^{(w)}$ is Morita equivalent to the incidence algebra of a lattice.

In Section 6, we derive (parts of) the representation theory of $M(W)$ from Sections 3, 4 , and 5

A long version of this paper with all proofs included will appear separately.

\section{Acknowledgments}

We would like to thank Jason Bandlow, Mahir Can, Brant Jones, Alain Lascoux, Jean-Christophe Novelli, Jean-Éric Pin, Vic Reiner, Franco Saliola, Benjamin Steinberg, and Jean-Yves Thibon for enlightening discussions. This research was driven by computer exploration, using the open-source mathematical software Sage [S $\left[\mathrm{S}^{+} 09\right]$ and its algebraic combinatorics features developed by the Sage-Comb inat community [SCc08]. AS and NT were in part supported by NSF grants DMS-0652641 and DMS-0652652. AS was in part supported by the Kastler foundation. FH was partly supported by ANR grant 06-BLAN-0380. 


\section{Blocks of Coxeter group elements and the cutting poset}

In this section, we develop the combinatorics underlying the representation theory of the translation modules studied in Section 5. The key question is: for which subsets $J \subseteq I$ does the canonical bijection between a Coxeter group $W$ and the Cartesian product $W_{J} \times{ }^{J} W$ of a parabolic subgroup $W_{J}$ by its set of coset representatives ${ }^{J} W$ in $W$ restrict properly to an interval $[1, w]_{R}$ in right order (see Figure 1 ? ? In type $A$, the answer is given by the so-called blocks in the permutation matrix of $w$, and we generalize this notion to any Coxeter group. After reviewing parabolic subgroups and cosets representatives in Section 2.1, we define blocks of Coxeter group elements in Section 2.2, and show in Section 2.3 how this notion specializes to type $A$. Finally, in Section 2.4. we introduce and study the cutting poset.

\subsection{Parabolic subgroups and cosets representatives}

For a subset $J \subseteq I$, the parabolic subgroup $W_{J}$ of $W$ is the Coxeter subgroup of $W$ generated by $s_{j}, j \in J$. A complete system of minimal length representatives of the right cosets $W_{J} w$ (resp. of the left cosets $w W_{J}$ ) are given respectively by:

$$
{ }^{J} W:=\left\{x \in W \mid \mathrm{D}_{L}(x) \cap J=\emptyset\right\} \quad \text { and } \quad W^{J}:=\left\{x \in W \mid \mathrm{D}_{R}(x) \cap J=\emptyset\right\} .
$$

Every $w \in W$ has a unique decomposition $w=w_{J}{ }^{J} w$ with $w_{J} \in W_{J}$ and ${ }^{J} w \in{ }^{J} W$. Similarly, there is a unique decomposition $w=w^{K}{ }_{K} w$ with ${ }_{K} w \in{ }_{K} W=W_{K}$ and $w^{K} \in W^{K}$. A subset $J$ is left reduced w.r.t. $w$ if $J^{\prime} \subset J$ implies ${ }^{J^{\prime}} w>{ }_{L}{ }^{J} w$. Right reduced $K$ 's are defined analogously.

\subsection{Blocks of Coxeter group elements}

We now come to the definition of blocks of Coxeter group elements, and associated cutting points.

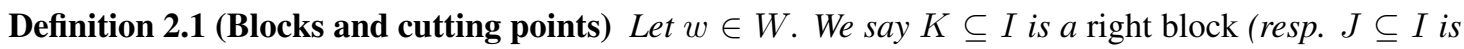
a left block) of $w$, if there exists $J \subseteq I$ (resp. $K \subseteq I$ ) such that $w W_{K}=W_{J} w$.

In that case, $v:=w^{K}$ is called a cutting point of $w$, which we denote by $v \sqsubseteq w$. Furthermore, $K$ is proper if $K \neq \emptyset$ and $K \neq I$; it is nontrivial if $w^{K} \neq w$ (or equivalently ${ }_{K} w \neq 1$ ); analogous definitions are made for left blocks.

We denote by $\mathcal{B}_{\mathcal{R}}(w)$ (resp. $\mathcal{B}_{\mathcal{L}}(w)$ ) the set of all right (resp. left) blocks for $w$, and by $\mathcal{R} \mathcal{B}_{\mathcal{R}}(w)$ (resp. $\mathcal{R B}_{\mathcal{L}}(w)$ ) the set of all reduced right (resp. left) blocks for $w$.

Proposition 2.2 Assuming that $J$ is reduced, $J$ is a left block of $w$ if and only if the bijection

$$
\begin{cases}W_{J} \times{ }^{J} W & \rightarrow W \\ (u, v) & \mapsto u v\end{cases}
$$

restricts to a bijection $\left[1, w_{J}\right]_{R} \times\left[1,{ }^{J} w\right]_{R} \rightarrow[1, w]_{R}$ (see Figure 1 ).

Due to Proposition 2.2. we also say that $[1, v]_{R}$ tiles $[1, w]_{R}$ if $v={ }^{J} w$ for some left block $J$.

Example 2.3 For $w=w_{0}$, any $K \subseteq I$ is a reduced right block; of course, $w_{0}^{K} \leq_{L} w_{0}$ and ${ }_{K} w_{0}$ is the maximal element of the parabolic subgroup $W_{K}={ }_{K} W$. The cutting point $w^{K} \sqsubseteq w$ is the maximal element of the right descent class for the complement of $K$. 
Proposition 2.4 The set $\mathcal{B}_{\mathcal{L}}(w)$ (resp. $\mathcal{B}_{\mathcal{R}}(w)$ ) of left (resp. right) blocks is stable under union and intersection. So, they form a sublattice of the Boolean lattice.

The sets $\mathcal{R B}_{\mathcal{L}}(w)$ and $\mathcal{R} \mathcal{B}_{\mathcal{R}}(w)$ are (dual) Moore families and therefore lattices.

Definition 2.5 ( $w$-codescent sets) For $u \in[1, w]_{R}$ define $K^{(w)}(u)$ to be the maximal reduced right block $K$ of $w$ such that $u$ is below the corresponding cutting point, that is $u \leq_{R} w^{K}$. Let $J^{(w)}(u)$ be the left block corresponding to $K^{(w)}(u)$.

Example 2.6 When $w=w_{0}$, any $J \subseteq I$ is a reduced left block. Furthermore, for $u \in W, J^{\left(w_{0}\right)}(u)$ is the complement of its left-descent set: $J^{\left(w_{0}\right)}(u)=I \backslash \mathrm{D}_{L}(u)$. Idem on the right.

\subsection{Blocks of permutations}

We now specialize to type $A_{n-1}$. For a permutation $w \in \mathfrak{S}_{n}$, the blocks of Definition 2.1 correspond to the usual notion of blocks of the matrix of $w$ (or unions thereof), and the cutting points $w^{K}$ for right blocks $K$ correspond to putting the identity in the matrix-blocks of $w$. Namely, a matrix-block of a permutation $w$ is an interval $\left[k^{\prime}, k^{\prime}+1, \ldots, k\right]$ which is mapped to another interval. Pictorially, this corresponds to a square submatrix of the matrix of $w$ which is again a permutation matrix (that of the associated permutation). For example, the interval $[2,3,4,5]$ is mapped to the interval $[4,5,6,7]$ by the permutation $w=36475812 \in \mathfrak{S}_{8}$, and is therefore a matrix-block of $w$ with associated permutation 3142. Similarly, $[7,8]$ is a matrix-block with associated permutation 12 :

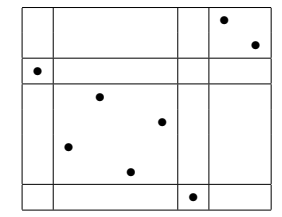

The singletons $[i]$ and the full set $[1,2, \ldots, n]$ are always matrix-blocks; the other matrix-blocks are called proper. A permutation with no proper matrix-block, such as 58317462 , is called simple. See [AA05] for a review of simple permutations or, equivalently, dimension 2 posets.

Proposition 2.7 Let $w \in \mathfrak{S}_{n}$. The right blocks of $w$ are in bijection with disjoint unions of (non singleton) matrix-blocks for $w$; each matrix-block with column set $[i, i+1, \ldots, k]$ contributes $\{i, i+1, \ldots, k-1\}$ to the right block; each matrix-block with row set $[i, i+1, \ldots, k]$ contributes $\{i, i+1, \ldots, k-1\}$ to the left block. In addition, trivial right blocks correspond to unions of identity matrix-blocks. Also, reduced right blocks correspond to unions of connected matrix-blocks.

Example 2.8 As in Figure 1$]$ consider the permutation 4312, whose permutation matrix is:

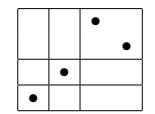

The reduced (right)-blocks are $K=\{\},\{1\},\{2,3\}$, and $\{1,2,3\}$. The cutting points are 4312,3412 , 4123, and 1234, respectively. The corresponding left blocks are $J=\{\},\{3\},\{1,2\}$ and $\{1,2,3\}$, respectively. The non-reduced blocks are $\{3\}$ and $\{1,3\}$, as they are respectively equivalent to the blocks \{\} and $\{1\}$. Finally, the trivial blocks are \{\} and $\{3\}$. 

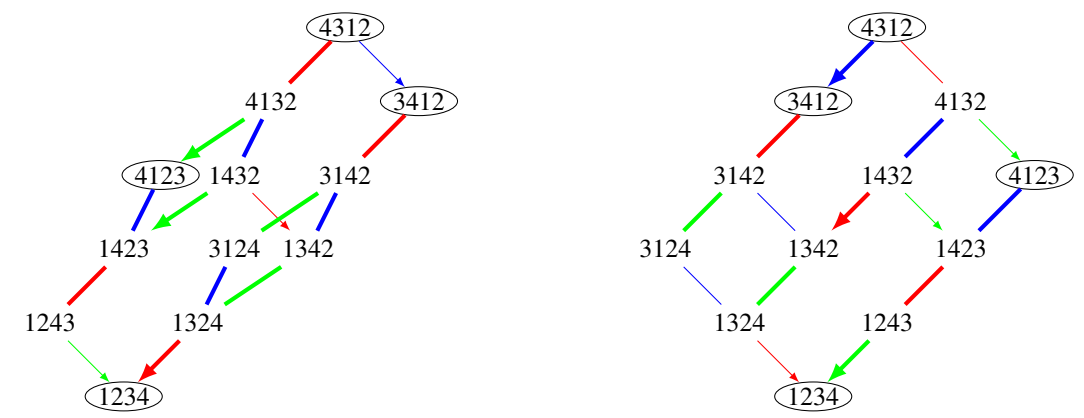

Fig. 1: Two pictures of the interval $[1234,4312]_{R}$ in right order illustrating its proper tilings, for $J:=\{3\}$ and $J:=\{1,2\}$, respectively. The thick edges highlight the tiling. The circled permutations are the cutting points, which are at the top of the tiling intervals. Blue, red, green lines correspond to $s_{1}, s_{2}, s_{3}$, respectively. See Section 5.4 for the definition of the orientation of the edges (this is $G^{(4312)}$ ); edges with no arrow tips point in both directions.

\subsection{The cutting poset}

Theorem $2.9(W, \sqsubseteq)$ is a poset with 1 as minimal element; it is further a subposet of both left and right order. Every interval of $(W, \sqsubseteq)$ is a sublattice of both left and right order.

The $\sqsubseteq$-lower covers of an element $w$ correspond to the nontrivial blocks of $w$ which are minimal for inclusion. The meet-semilattice $L_{w}$ they generate is free, and is in correspondence with the lattice of unions of these minimal nontrivial blocks, or alternatively of the intersections of the intervals $[1, u]_{R}$ for $u \sqsubseteq$-lower covers of $w$.

The Möbius function is given by $\mu(u, w)= \pm 1$ if $u$ is in $L_{w}$ (with alternating sign according to the usual rule for the Boolean lattice), and 0 otherwise.

This Möbius function is used in Section 5.4 to compute the size of the simple modules of $M$.

Conjecture 2.10 $(W, \sqsubseteq)$ is a meet-semilattice whose intervals are all distributive lattices.

\section{The combinatorics of $M(W)$}

In this section we study the combinatorics of the biHecke monoid $M(W)$ of a finite Coxeter group $W$. In particular, we prove in Section 3.1 that its elements preserve left order and Bruhat order, and derive in Section 3.2 properties of their image sets and fibers. Finally, in Section 3.3, we prove the key combinatorial ingredients for the enumeration of the simple modules of $M(W)$ in Section $6 M(W)$ is aperiodic and admits $|W|$ conjugacy classes of idempotents.

\subsection{Preservation of left and Bruhat order}

Lemma 3.1 Take $f \in M(W), w \in W$, and $j \in I$. Then, $\left(s_{j} w\right)$. f is either $w . f$ or $s_{j}(w . f)$.

Proposition 3.2 The elements $f$ of $M$ preserve left order: $u \leq_{L} v \Rightarrow u . f \leq_{L} v . f$.

Proposition 3.3 The elements $f$ of $M$ preserve Bruhat order: $u \leq_{B} v \Rightarrow u . f \leq_{B} v . f$.

Proposition 3.4 Any $f \in M$ such that $1 . f=1$ is contracting for Bruhat order: $w . f \leq_{B} w$. 


\subsection{Fibers and image sets}

Proposition 3.5 The image set of an idempotent in $M(W)$ is an interval in left order.

Proposition 3.6 Take $f \in M(W)$, and consider the Hasse diagram of left order contracted with respect to the fibers of $f$. Then, this graph is isomorphic to left order restricted on the image set.

Proposition 3.7 Any element $f \in M(W)$ is characterized by its set of fibers and its image set.

A monoid $M$ is called aperiodic if for any $f \in M$, there exists $k>0$ such that $f^{k+1}=f^{k}$. Note that, in that case, $f^{\infty}:=f^{k}=f^{k+1}=\ldots$ is an idempotent.

Proposition 3.8 The biHecke monoid $M(W)$ is aperiodic.

\subsection{Conjugacy classes of idempotents}

Proposition 3.9 For $w \in W, e_{w}:=\pi_{w^{-1} w_{0}} \bar{\pi}_{w_{0} w}$ is the unique idempotent with image set $[1, w]_{L}$. For $u \in W$, it satisfies $e_{w}(u)=\max _{\leq_{B}}\left([1, u]_{B} \cap[1, w]_{L}\right)$.

Corollary 3.10 For $u, w \in W$, the intersection $[1, u]_{B} \cap[1, w]_{L}$ is a lower $\leq_{L}$ ideal with a unique maximal element $v$ in Bruhat order. The maximum is given by $v=e_{w}(u)$.

We are now in the position to describe the conjugacy relations between the idempotents of $M$.

Lemma 3.11 Let $e$ and $f$ be idempotents with respective image sets $[a, b]_{L}$ and $[c, d]_{L}$. Then, $f \in M e M$ if and only if $d c^{-1} \leq_{L R} b a^{-1}$. In particular, $e$ and $f$ are conjugates if and only if the intervals $[a, b]_{L}$ and $[c, d]_{L}$ are of the same type: $d c^{-1}=b a^{-1}$.

Corollary 3.12 The idempotents $\left(e_{w}\right)_{w \in W}$ form a complete set of representatives of the conjugacy classes of idempotents in $M$.

\section{The Borel submonoid $M_{1}(W)$ and its representation theory}

In the previous section, we outlined the importance of the idempotents $\left(e_{w}\right)_{w \in W}$. A crucial feature is that they live in a "Borel" submonoid $M_{1}:=\{f \in M \mid 1 . f=1\}$. In fact:

Theorem 4.1 $M_{1}$ has a unique minimal generating set which consists of the $\left(2^{n}-n\right.$ in type A) idempotents $e_{w}$ where $w_{0} w^{-1}$ is Grassmanian.

Furthermore, the elements of $M_{1}$ are both order-preserving and contracting for Bruhat order.

Corollary 4.2 For $f, g \in M_{1}$, define the relation $f \leq g$ if $w . f \leq w . g$ for all $w \in W$. Then, $\leq$ defines $a$ partial order on $M_{1}$ such that $f g \leq f$ and $f g \leq g$ for any $f, g \in M_{1}$.

$M_{1}$ is therefore $\mathcal{J}$-trivial (see e.g. [Pin09]). The generalization of most of the representation theoretical results summarized below to any $\mathcal{J}$-trivial monoid is the topic of [DHST10].

For each $w \in W$ define $S_{w}$ to be the one-dimensional vector space with basis $\left\{\epsilon_{w}\right\}$ together with the right operation of any $f \in M_{1}$ given by $\epsilon_{w} . f:=\epsilon_{w}$ if $w . f=w$ and $\epsilon_{w} . f:=0$ otherwise. The basic features of the representation theory of $M_{1}$ can be stated as follows:

Theorem 4.3 The radical of $\mathbb{K}\left[M_{1}\right]$ is the ideal with basis $\left(f^{\infty}-f\right)_{f}$, for $f$ non-idempotent. The quotient of $\mathbb{K}\left[M_{1}\right]$ by its radical is commutative. Therefore, all simple $M_{1}$-module are one dimensional. In fact, the family $\left\{S_{w}\right\}_{w \in W}$ forms a complete system of representatives of the simple $M_{1}$-modules. 
To describe the indecomposable projective modules, we note that the restriction of the conjugacy relation ( $\mathcal{J}$-order) to idempotents has a very simple description:

Proposition 4.4 For $u, v \in W$, the following are equivalent:

$$
\begin{array}{ll}
\bullet e_{u} e_{v}=e_{u} ; & \bullet v \leq_{L} \text { u for left order; } \\
\bullet e_{v} e_{u}=e_{u} ; & \bullet \text { there exists } x, y \in M_{1} \text { such that } e_{u}=x e_{v} y .
\end{array}
$$

Moreover $\left(e_{u} e_{v}\right)^{\infty}=e_{u \vee_{L} v}$, where $u \vee_{L} v$ is the join of $u$ and $v$ in left order.

Definition 4.5 For any element $x \in M$, define

$$
\operatorname{lfix}(x):=\min _{\leq_{L}}\left\{u \in W \mid e_{u} x=x\right\} \quad \text { and } \quad \operatorname{rfix}(x):=\min _{\leq_{L}}\left\{u \in W \mid x e_{u}=x\right\}
$$

Then, the projective modules and Cartan invariants can be described as follows:

Theorem 4.6 There is an explicit basis $\left(b_{f}\right)_{f \in M_{1}}$ of $\mathbb{K}\left[M_{1}\right]$ such that, for all $w \in W$,

- the family $\left\{b_{x} \mid \operatorname{lfix}(x)=w\right\}$ is a basis for the right projective module associated to $S_{w}$;

- the family $\left\{b_{x} \mid \operatorname{rfix}(x)=w\right\}$ is a basis for the left projective module associated to $S_{w}$.

Moreover, the Cartan invariant of $\mathbb{K}\left[M_{1}\right]$ defined by $c_{u, v}:=\operatorname{dim}\left(e_{u} \mathbb{K}\left[M_{1}\right] e_{v}\right)$ for $u, v \in W$ is given by $c_{u, v}=\left|C_{u, v}\right|$, where $C_{u, v}:=\left\{f \in M_{1} \mid u=\operatorname{lfix}(f)\right.$ and $\left.v=\operatorname{rfix}(f)\right\}$.

\section{Translation modules and w-biHecke algebras}

The main purpose of this section is to pave the ground for the construction of the simple modules $S_{w}$ of the biHecke monoid $M=M(W)$ in Section 6 To this end, in Section 5.1, we endow the interval $[1, w]_{R}$ with a natural structure of a combinatorial $M$-module $T_{w}$, called translation module. This module is closely related to the projective module $P_{w}$ of $M$ (Corollary 6.2), which explains its important role. By taking the quotient of $\mathbb{K}[M]$ through its representation on $T_{w}$, we obtain a $w$-analogue $\mathcal{H} W^{(w)}$ of the biHecke algebra $\mathcal{H} W$. This algebra turns out to be interesting in its own right, and we proceed by generalizing most of the results of [HT09] on the representation theory of $\mathcal{H} W$.

As a first step, we introduce in Section 5.2 a collection of submodules $P_{J}^{(w)}$ of $T_{w}$, which are analogues of the projective modules of $\mathcal{H} W$. Unlike for $\mathcal{H} W$, not any subset $J$ of $I$ yields such a submodule, and this is where the combinatorics of the blocks of $w$ as introduced in Section 2 enters the game. In a second step, we derive in Section 5.3 a lower bound on the dimension of $\mathcal{H} W^{(w)}$; this requires a (fairly involved) combinatorial construction of a family of functions on $[1, w]_{R}$ which is triangular with respect to Bruhat order. In Section 5.4 we combine these results to derive the dimension and representation theory of $\mathcal{H} W^{(w)}$ : projective and simple modules, Cartan matrix, quiver, etc.

\subsection{Translation modules and w-biHecke algebras}

For $f \in M$, define the type of $f$ by type $(f):=\left(w_{0} \cdot f\right)(1 . f)^{-1}$. By Proposition 3.2 we know that for $f, g \in M$ either type $(f g)=\operatorname{type}(f)$, or $\ell\left(w_{0} \cdot(f g)\right)-\ell(1 .(f g))<\ell\left(w_{0} \cdot f\right)-\ell(1 . f)$ and hence type $(f g) \neq \operatorname{type}(f)$. The second case occurs precisely when fiber $(f)$ is strictly finer than fiber $(f g)$ or equivalently $\operatorname{rank}(f g)<\operatorname{rank}(f)$, where the rank is the cardinality of the image set. 
Definition 5.1 Fix $f \in M$. The right $M$-module

$$
\operatorname{trans}(f):=\mathbb{K} . f M / \mathbb{K} .\{h \in f M \mid \operatorname{rank}(h)<\operatorname{rank}(f)\}
$$

is called the translation module associated with $f$.

Proposition 5.2 Fix $f \in M$. Then:

$$
\{h \in f M \mid \operatorname{rank}(h)=\operatorname{rank}(f)\}=\left\{f_{u} \mid u \in\left[1, \operatorname{type}(f)^{-1} w_{0}\right]_{R}\right\},
$$

where $f_{u}$ is the unique element of $M$ such that $\operatorname{fiber}\left(f_{u}\right)=\operatorname{fiber}(f)$ and $1 . f_{u}=u$.

Proposition 5.3 Set $w=\operatorname{type}(f)^{-1} w_{0}$. Then, $\left(f_{u}\right)_{u \in[1, w]_{R}}$ forms a basis of $\operatorname{trans}(f)$ such that:

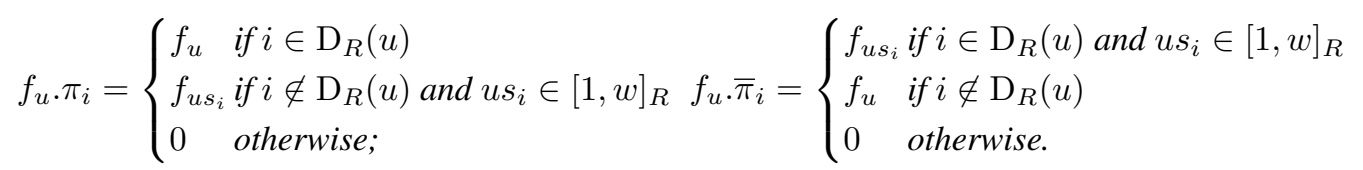

This proposition gives a combinatorial model for translation modules. It is clear that two functions with the same type yield isomorphic translation modules. The converse also holds:

Proposition 5.4 For any $f, f^{\prime} \in M$, the translation modules $\operatorname{trans}(f)$ and $\operatorname{trans}\left(f^{\prime}\right)$ are isomorphic if and only if type $(f)=\operatorname{type}\left(f^{\prime}\right)$.

By the previous proposition, we may choose a canonical representative for translation modules. We choose $T_{w}:=\operatorname{trans}\left(e_{w, w_{0}}\right)$, and identify its basis with $[1, w]_{R}$ via $u \mapsto f_{u}$.

Definition 5.5 The $w$-biHecke algebra $\mathcal{H} W^{(w)}$ is the natural quotient of $\mathbb{K}[M(W)]$ through its representation on $T_{w}$. In other words, it is the subalgebra of $\operatorname{End}\left(T_{w}\right)$ generated by the operators $\pi_{i}$ and $\bar{\pi}_{i}$ of Proposition 5.3

\subsection{Left antisymmetric submodules}

By analogy with the simple reflections in the Hecke group algebra, we define for each $i \in I$ the operator $s_{i}:=\pi_{i}+\bar{\pi}_{i}-1$. For $u \in[1, w]_{R}$, it satisfies $u . s_{i}=u s_{i}$ if $u s_{i} \in[1, w]_{R}$ and $u . s_{i}=-u$ otherwise. These operators are still involutions, but do not quite satisfy the braid relations. One can further define operators $\overleftarrow{s}_{i}$ acting similarly on the left.

Definition 5.6 For $J \subseteq I$, set $P_{J}^{(w)}:=\left\{v \in T_{w} \mid \overleftarrow{s}_{i} \cdot v=-v, \quad \forall i \in J\right\}$

For $w=w_{0}$, these are the projective modules $P_{J}$ of the biHecke algebra.

Proposition 5.7 Take $w \in W$ and $J \subseteq I$ left reduced. Then, $J$ is a reduced left block of $w$ if and only if $P_{J}^{(w)}$ is a submodule of $T_{w}$.

It is clear from the definition that for $J_{1}, J_{2} \subseteq I, P_{J_{1} \cup J_{2}}^{(w)}=P_{J_{1}}^{(w)} \cap P_{J_{2}}^{(w)}$. Since the set $\mathcal{R} \mathcal{B}_{\mathcal{L}}(w)$ of reduced left blocks of $w$ is stable under union, the set of modules $\left(P_{J}^{(w)}\right)_{J \in \mathcal{R} \mathcal{B}_{\mathcal{L}}(w)}$ is stable under intersection. On the other hand, unless $J_{1}$ and $J_{2}$ are comparable, $P_{J_{1} \cup J_{2}}^{(w)}$ is a strict subspace of $P_{J_{1}}^{(w)}+$ $P_{J_{2}}^{(w)}$. Hence, for $J \in \mathcal{R} \mathcal{B}_{\mathcal{L}}(w)$, we set $S_{J}^{(w)}:=P_{J}^{(w)} / \sum_{J^{\prime} \supsetneq J, J^{\prime} \in \mathcal{R} \mathcal{B}_{\mathcal{L}}(w)} P_{J^{\prime}}^{(w)}$. 


\subsection{A (maximal) Bruhat-triangular family of $\mathcal{H} W^{(w)}$}

Consider the submonoid $F$ in $\mathcal{H} W^{(w)}$ generated by the operators $\pi_{i}, \bar{\pi}_{i}$, and $s_{i}$, for $i \in I$. For $f \in F$ and $u \in[1, w]_{R}$, we have $u . f= \pm v$ for some $v \in[1, w]_{R}$. For our purposes, the signs can be ignored and $f$ be considered as a function from $[1, w]_{R}$ to $[1, w]_{R}$.

Definition 5.8 For $u, v \in[1, w]_{R}$, a function $f \in F$ is called $(u, v)$-triangular (for Bruhat order) if $v$ is the unique minimal element of $\operatorname{im}(f)$ and $u$ is the unique maximal element of $f^{-1}(v)$ (all minimal and maximal elements in this context are with respect to Bruhat order).

Proposition 5.9 Take $u, v \in[1, w]_{R}$ such $K^{(w)}(u) \subseteq K^{(w)}(v)$. Then, there exists a $(u, v)$-triangular function $f_{u, v}$ in $F$.

For example, for $w=4312$ in $\mathfrak{S}_{4}$, the condition on $u$ and $v$ is equivalent to the existence of a path from $u$ to $v$ in the digraph $G^{(4312)}$ (see Figure 1 and Section 5.4.

The construction of $f_{u, v}$ is explicit, and the triangularity derives from $f_{u, v}$ being either in $M$, or close enough to be bounded below by an element of $M$. It follows from the upcoming Theorem 5.10 that the condition on $u$ and $v$ is not only sufficient but also necessary.

\subsection{Representation theory of $w$-biHecke algebras}

Consider the digraph $G^{(w)}$ on $[1, w]_{R}$ with an edge $u \mapsto v$ if $u=v s_{i}$ for some $i$, and $J^{(w)}(u) \subseteq J^{(w)}(v)$. Up to orientation, this is the Hasse diagram of right order (see for example Figure 11). The following theorem is a generalization of [HT09, Section 3.3].

Theorem 5.10 $\mathcal{H} W^{(w)}$ is the maximal algebra stabilizing all the modules $P_{J}^{(w)}$, for $J \in \mathcal{R} \mathcal{B}_{\mathcal{L}}(w)$.

The elements $f_{u, v}$ of Proposition 5.9 form a basis $\mathcal{H} W^{(w)}$; in particular,

$$
\operatorname{dim} \mathcal{H} W^{(w)}=\left|\left\{(u, v) \mid J^{(w)}(u) \subseteq J^{(w)}(v)\right\}\right| .
$$

$\mathcal{H} W^{(w)}$ is the digraph algebra of the graph $G^{(w)}$.

The family $\left(P_{J}\right)_{J \in \mathcal{R} \mathcal{B}_{\mathcal{L}}(w)}$ forms a complete system of representatives of the indecomposable projective modules of $\mathcal{H} W^{(w)}$.

The family $\left(S_{J}\right)_{J \in \mathcal{R} \mathcal{B}_{\mathcal{L}}(w)}$ forms a complete system of representatives of the simple modules of $\mathcal{H} W^{(w)}$. The dimension of $S_{J}$ is the size of the corresponding w-descent class.

$\mathcal{H} W^{(w)}$ is Morita equivalent to the poset algebra of the lattice $[1, w]_{\sqsubseteq}$.

\section{The representation theory of $M(W)$}

Theorem 6.1 The monoid $M=M(W)$ admits $|W|$ non-isomorphic simple modules $\left(S_{w}\right)_{w \in W}$ (resp. projective indecomposable modules $\left.\left(P_{w}\right)_{w \in W}\right)$.

The simple module $S_{w}$ is isomorphic to the top simple module $S_{\{\}}^{(w)}$ of the translation module $T_{w}$. In general, the simple quotient module $S_{J}^{(w)}$ of $T_{w}$ is isomorphic to $S_{J_{w}}$ of $M$.

For example, the simple module $S_{4312}$ is of dimension 3, with basis $\{4312,4132,1432\}$ (see Figure 1 ). The other simple modules $S_{3412}, S_{4123}$, and $S_{1234}$ are of dimension 5,3 , and 1 .

Corollary 6.2 The translation module $T_{w}$ is an indecomposable $M$-module, quotient of the projective module $P_{w}$ of $M$. 
$M_{1}$ is a submonoid of $M$. Therefore any $M$-module $X$ is a $M_{1}$-module, and its $M_{1}$-character $[X]_{M_{1}}$ depends only on its $M$-character $[X]_{M}$. This defines a $\mathbb{Z}$-linear map $[X]_{M} \mapsto[X]_{M_{1}}$. Let $\left(S_{w}\right)_{w \in W}$ and $\left(S_{w}^{1}\right)_{w \in W}$ be complete families of simple modules representatives for $M$ and $M_{1}$, respectively. The matrix of $[X]_{M} \mapsto[X]_{M_{1}}$ is called the decomposition matrix of $M$ over $M_{1}$; its coefficient $(u, v)$ is the multiplicity of $S_{u}^{1}$ as a composition factor of $S_{v}$ viewed as an $M_{1}$-module.

Theorem 6.3 The decomposition matrix of $M$ over $M_{1}$ is upper uni-triangular for right order, with 0,1 entries. Furthermore, $T_{w}$ is isomorphic to the induction to $M$ of the simple module $S_{w}^{1}$.

\section{References}

[AA05] M. H. Albert and M. D. Atkinson. Simple permutations and pattern restricted permutations. Discrete Math., 300(1-3):1-15, 2005.

[BB05] A. Björner and F. Brenti. Combinatorics of Coxeter groups, volume 231 of Graduate Texts in Mathematics. Springer, New York, 2005.

[DHST10] T. Denton, F. Hivert, A. Schilling, and N. M. Thiéry. The representation theory of $\mathcal{J}$-trivial monoids. In preparation, 2010.

[Ges84] I. M. Gessel. Multipartite $P$-partitions and inner products of skew Schur functions. In Combinatorics and algebra (Boulder, Colo., 1983), volume 34 of Contemp. Math., pages 289-317. Amer. Math. Soc., Providence, RI, 1984.

[HST09] F. Hivert, A. Schilling, and N. M. Thiéry. Hecke group algebras as quotients of affine Hecke algebras at level 0. J. Combin. Theory Ser. A, 116(4):844-863, 2009.

[HT09] F. Hivert and N. M. Thiéry. The Hecke group algebra of a Coxeter group and its representation theory. J. Algebra, 321(8):2230-2258, 2009.

[Hum90] J. E. Humphreys. Reflection groups and Coxeter groups, volume 29 of Cambridge Studies in Advanced Mathematics. Cambridge University Press, Cambridge, 1990.

[KT97] D. Krob and J.-Y. Thibon. Noncommutative symmetric functions. IV. Quantum linear groups and Hecke algebras at $q=0$. J. Algebraic Combin., 6(4):339-376, 1997.

[Pin09] J.-É. Pin. Mathematical grounds of automata theory. 2009.

$\left[\mathrm{S}^{+} 09\right]$ W. A. Stein et al. Sage Mathematics Software (Version 3.3). The Sage Development Team, 2009. http://www. sagemath.org.

[SCc08] The Sage-Combinat community. Sage-Combinat: enhancing sage as a toolbox for computer exploration in algebraic combinatorics, 2008. http: // combinat. sagemath.org. 